\title{
A mobility study in commercial and industrial areas of Stuttgart - Experiences and conclusions promoting intermodality of commuters
}

Günter Sabow

Wirtschafts- und Industrievereinigung Stuttgart e.V.

This manuscript is not available according to publishing restriction. Thank you for your understanding. 